\title{
Electronic structure, cohesive, and magnetic properties of the actinide-iridium Laves phases
}

Eriksson, O.; Johansson, B.; Brooks, M. S. S.; Skriver, Hans Lomholt

Published in:

Physical Review B

Link to article, DOI:

10.1103/PhysRevB.39.5647

Publication date:

1989

Document Version

Publisher's PDF, also known as Version of record

Link back to DTU Orbit

Citation (APA):

Eriksson, O., Johansson, B., Brooks, M. S. S., \& Skriver, H. L. (1989). Electronic structure, cohesive, and magnetic properties of the actinide-iridium Laves phases. Physical Review B, 39(9), 5647-5654.

https://doi.org/10.1103/PhysRevB.39.5647

\section{General rights}

Copyright and moral rights for the publications made accessible in the public portal are retained by the authors and/or other copyright owners and it is a condition of accessing publications that users recognise and abide by the legal requirements associated with these rights.

- Users may download and print one copy of any publication from the public portal for the purpose of private study or research.

- You may not further distribute the material or use it for any profit-making activity or commercial gain

- You may freely distribute the URL identifying the publication in the public portal 


\title{
Electronic structure, cohesive, and magnetic properties of the actinide-iridium Laves phases
}

\author{
O. Eriksson \\ Condensed Matter Theory Group, Department of Physics, University of Uppsala, Box 530, S-75121 Uppsala, Sweden \\ B. Johansson* \\ Center for Materials Science, Los Alamos National Laboratory, Los Alamos, New Mexico 87545 \\ M. S. S. Brooks \\ Commission of the European Communities, Joint Research Centre, European Institute for Transuranium Elements, \\ Postfach 2340, D-7500 Karlsruhe, Federal Republic of Germany \\ H. L. Skriver \\ Laboratory of Applied Physics, Technical University of Denmark, DK-2800 Lyngby, Denmark
}

(Received 3 October 1988)

\begin{abstract}
The electronic structure of the isostructural $A \operatorname{Ir}_{2}$ systems ( $A=\mathrm{Th}, \mathrm{Pa}, \mathrm{U}, \mathrm{Np}, \mathrm{Pu}$, and $\mathrm{Am}$ ) has been obtained by means of the scalar relativistic and fully relativistic linear muffin-tin orbital techniques. Ground-state properties such as lattice constants and onset of magnetic order have been calculated and compared with measured data. The hybridization between the actinide $5 f$ and the ligand $5 d$ states and the direct $5 f$ wave-function overlap are found to be of comparable importance for the bandwidth of the itinerant $5 f$ states. The anomalous paramagnetism of $\mathrm{PuIr}_{2}$ can be explained only by a fully relativistic treatment.
\end{abstract}

\section{INTRODUCTION}

The $A \operatorname{Ir}_{2}(A=\mathrm{Th}, \mathrm{Pa}, \mathrm{U}, \mathrm{Np}, \mathrm{Pu}, \mathrm{Am}$, and $\mathrm{Cm})$ series of intermetallic compounds exhibits many interesting physical properties. It is found that $\operatorname{ThIr}_{2}$ becomes superconducting below $6.5 \mathrm{~K}^{1} \mathrm{UIr}_{2}$ is a nearly temperature-independent paramagnet, with a small maximum in the magnetic susceptibility at $60 \mathrm{~K}^{2}$ The coefficient of the linear contribution to the specific heat $\gamma$ is high $\left(62 \mathrm{~mJ} / \mathrm{mol} \mathrm{K}^{2}\right.$ ) (Ref. 3) and the resistivity shows a $T^{2}$ dependence at low temperatures. ${ }^{2}$ These two facts have been taken to be an indication of spin-fluctuation effects in $\mathrm{UIr}_{2} .{ }^{2} \mathrm{NpIr}_{2}$ is the first compound in the $A \mathrm{Ir}_{2}$ series to order magnetically. Mössbauer experiments indicate that it is an antiferromagnet with an ordering temperature of $5.8 \mathrm{~K}$ (Ref. 4 ) or $7.5 \mathrm{~K}$ (Ref. 5) and the measured hyperfine field corresponds to a magnetic moment of $0.6 \mu_{B} \cdot^{4,5} \mathrm{~A}$ maximum in the susceptibility was found at $7.5 \mathrm{~K},{ }^{5}$ but the specific heat showed a maximum at a somewhat lower temperature, indicating that the Neél temperature is $6.6 \mathrm{~K} .^{6}$ The small moment together with a low magnetic entropy of $0.38 R \ln 2$ suggest that $\mathrm{NpIr}_{2}$ is an itinerant antiferromagnet. ${ }^{5,6}$ The measured coefficient of the linear contribution to the specific heat $\gamma$ is very high in the antiferromagnetic phase $\left(400 \mathrm{~mJ} / \mathrm{mol} \mathrm{K}^{2}\right)$ and an extrapolation for the paramagnetic phase gives a $\gamma$ value of $234 \mathrm{~mJ} / \mathrm{mol} \mathrm{K}{ }^{2}$. The next compound $\mathbf{P u I r}_{2}$ is a temperature-independent paramagnet ${ }^{7,8}$ and the resistivity shows a temperature dependence of the form $\rho=\rho_{0}+B T^{3}$. The $T^{3}$ dependence was argued to be characteristic of $s-f$ interband scattering. ${ }^{7}$ Finally, to the authors knowledge, only the lattice constants are known for $\mathrm{AmIr}_{2}$ and $\mathrm{CmIr}_{2}$.

Since the $A \operatorname{Ir}_{2}$ systems are isostructural and crystallize in the relatively simple, cubic Laves phase (C15), they are attractive for theoretical studies. Here as in many other intermetallic compounds involving $5 f$ states, there is strong evidence that the $5 f$ electrons play a crucial role both for the chemical bonding ${ }^{9}$ and the magnetic properties. ${ }^{10}$ The paramagnetic behavior of $\mathrm{PuIr}_{2}$ appears particularly anomalous since the preceding system $\mathrm{NpIr}_{2}$ is an antiferromagnet and $\mathrm{UIr}_{2}$ shows spin-fluctuation effects. Conventional ideas about $5 f$ band narrowing as one proceeds along the actinide series would suggest even more pronounced magnetism for $\mathrm{PuIr}_{2}$ than for $\mathrm{NpIr}_{2}$.

In the present work we report on $a b$ initio energy-band calculations for the $A \mathrm{Ir}_{2}$ series. We have performed both paramagnetic and spin-polarized equation of state calculations in order to elucidate the nature of the chemical bonding and the magnetism in the $A \mathrm{Ir}_{2}$ systems. Special attention was paid to the anomalous nonmagnetic state of $\mathrm{PuIr}_{2}$. To understand its properties we have performed fully relativistic calculations and have found it illustrative to compare these calculations with similar calculations for $\mathrm{UIr}_{2}$ and $\mathrm{NpIr}_{2}$. The role of the $5 f$ bonding in these systems has also been studied in more detail using a simplified model of the densities of states and this model can also serve as a basis for the understanding of the role of hybridization of the actinide $5 f$ states with the Ir $5 d$ states in these systems.

The problem of localized versus itinerant $5 f$ electrons in actinide metals and compounds is an old and fascinating one, and we will here consider this problem for the $A \mathrm{Ir}_{2}$ systems drawing special attention to the bonding nature of itinerant $5 f$ electrons. In the formation of $5 f$ bonds in actinide compounds hybridization has been shown to be of crucial importance ${ }^{11-15}$ as has the influence of the direct overlap between the $5 f$ wave func- 
tions. ${ }^{16}$ The latter effect forms the basis for the interpretation of the so-called Hill plots. ${ }^{17}$ For all $A \operatorname{Ir}_{2}$ systems up to $\mathrm{AmIr}_{2}$ the $A$ - $A$ spacing is lower than, or at the border of, the Hill limit for $5 f$ localization indicating that the $5 f$ electrons are itinerant for all the $A \mathrm{Ir}_{2}$ compounds with the exception of $\mathrm{AmIr}_{2}$ and $\mathrm{CmIr}_{2}$ (and the compounds beyond these two), in which they are localized.

In the computation of the electronic structure of the $A \mathrm{Ir}_{2}$ compounds, scalar and fully relativistic linear muffin-tin orbital energy-band calculations in the atomic-sphere approximation with the combined correction terms ${ }^{18}$ were performed. The frozen core approximation was used and the valence charge density was iterated until it was self-consistent with a maximum error in the valence charge density being of the order of one part into $10^{3}$ at each point of the radial mesh $\left(\Delta \rho<10^{-3} \rho, \rho\right.$ denotes density). The local spin-density approximation, with the von Barth-Hedin exchange and correlation potential ${ }^{19}$ was adopted. The energy bands were first calculated at $20 k$ points in the irreducible wedge of the Brillouin zone (BZ) and then for the final convergence at $89 k$ points. The energy bands and the ground-state properties (pressure, occupation number, and magnetic moment) were almost the same for these two calculations. Lattice parameters were obtained from the zero-temperature equation of state calculation. ${ }^{20}$

In Sec. II we present our results of the cohesive properties and in Sec. III we report on spin-polarized calculations. Section IV contains results of relativistic calculations and in Sec. $V$ we present a model calculation for $\mathrm{UIr}_{2}$. Conclusions are given in Sec. VI.

\section{COHESIVE PROPERTIES}

According to Vegard's law the volume of the unit cell of the compound is obtained by an appropriate sum of the metal atom volumes. ${ }^{21}$ We have plotted these expected compound volumes together with the measured ones in Fig. 1. Although Vegard's law is often obeyed, here the disagreement is apparent. The reason for this is twofold.

(a) It is for the compounds where we expect no or little $5 f$ bonding, i.e., $\mathrm{ThIr}_{2}, \mathrm{AmIr}_{2}$, and $\mathrm{CmIr}_{2}$, that the mea-

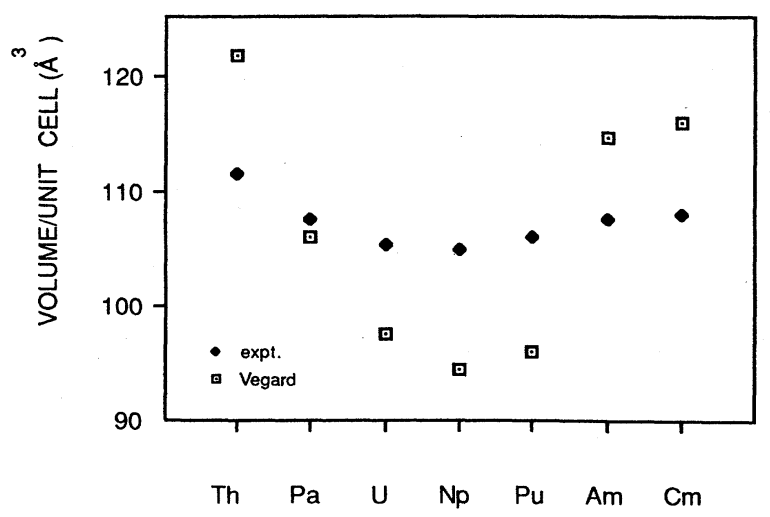

FIG. 1. Experimental volumes and volumes calculated from Vegard's law for the $A \mathrm{Ir}_{2}$ systems. sured volume is lower than predicted by Vegard's law. This reflects the large-energy gain due to the formation of the compound. This compound bonding contracts the lattice and the effect is largest for the formation of compounds between late and early transition metals, where the bonding orbitals can be filled and the antibonding orbitals left empty. For $\mathrm{ThIr}_{2}$ this volume contraction is 9.9 $\AA^{3}$ and for $\mathrm{AmIr}_{2}$ and $\mathrm{CmIr}_{2}$ it is about 8.0 $\AA^{3}$. The number of $d$ electrons for Ir is 7.4 and $\mathrm{Th}$, being tetravalent, has $1.9 d$ electrons whereas $\mathrm{Am}$ and $\mathrm{Cm}$ have 1.2 $d$ electrons each. Hence, upon formation of the compound, $\mathrm{ThIr}_{2}$ can with $2 \times 7.4+1.9=16.7 d$ electrons nearly fill its bonding $d$ band (which has the capacity to contain 20 electrons) and in $\mathrm{AmIr}_{2}$ and $\mathrm{CmIr}_{2}$, with 16.0 $d$ electrons, the bonding $d$ band is also nearly full. The volume contraction effect should, however, be a little less here, as compared to $\mathrm{ThIr}_{2}$, and this is indeed so.

(b) In sharp contrast to this, for the systems with a strong indication of having bonding $5 f$ electrons, the experimental volumes exceed Vegard's law volumes. We explain this apparent paradox by the difference between the $5 f$ bonding in the pure actinide metal and the corresponding $5 f$ bonding in the intermetallic compound. The electronic pressure the $5 f$ electrons contribute, can, in the simplest approximation, be divided into two components. ${ }^{20,22,12,9}$ One is called the band center term and is in the actinide metals and in the $A \operatorname{Ir}_{2}$ systems repulsive. The other term which is called the bandwidth term is negative (bonding) and dominates in these systems. It can, in its simplest form, be written

$$
(3 p V)_{f}=-n_{f}\left(14-n_{f}\right) \frac{W}{28}\left(-\frac{\partial \ln W}{\partial \ln S}\right),
$$

where $W$ is the bandwidth, $S$ is the Wigner-Seitz radius, and $n_{f}$ is the partial $5 f$ occupation number. As has been noted elsewhere ${ }^{23}$ the number of $5 f$ electrons is about the same in the metal as in an intermetallic compound (Table I), and an estimate of $-\delta \ln W / \delta \ln S$ is $2 l+1=7$ for $f$ states. ${ }^{12}$ Therefore the $5 f$ pressure will change, when going from a metal to a compound, only if the bandwidth $W$ changes. For example, we calculate the $5 f$ bandwidth in $\mathrm{UIr}_{2}$ to be $0.2 \mathrm{Ry}$ whereas it is $0.3 \mathrm{Ry}$ in uranium metal.

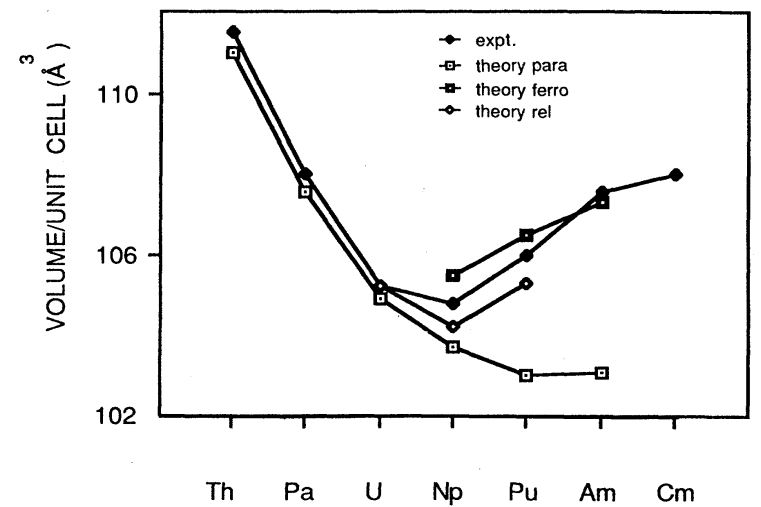

FIG. 2. Experimental and theoretical (scalar relativistic, spin-polarized scalar relativistic, and fully relativistic) volumes for the $A \mathrm{Ir}_{2}$ systems. 
TABLE I. Self-consistently calculated actinide $5 f$ occupation numbers, $n_{f}$; magnetic moments, $m_{\text {tot }}$; multiband Stoner products, $I D$; relativistic Stoner product, $I D$ (relativistic); and band masses, $\mu_{f}$, for the $A \operatorname{Ir}_{2}$ systems. Also given are the band center term, $(p V)_{C}$, and the bandwidth term, $(p V)_{W}$, from Eq. (2).

\begin{tabular}{lrrrrrr}
\hline \hline & $\mathrm{ThIr}_{2}$ & $\mathrm{PaIr}_{2}$ & $\mathrm{UIr}_{2}$ & $\mathrm{NpIr}_{2}$ & $\mathrm{PuIr}_{2}$ & $\mathrm{AmIr}_{2}$ \\
\hline$n_{f}$ & 0.8 & 1.7 & 2.9 & 4.0 & 5.2 & 6.3 \\
$(3 p V)_{C}(\mathrm{Ry})$ & 0.21 & 0.10 & 0.07 & 0.06 & 0.05 & 0.04 \\
$(3 p V)_{W}(\mathrm{Ry})$ & -1.06 & -1.08 & -1.15 & -1.10 & -0.96 & -0.87 \\
$I D$ & 0.15 & 0.22 & 0.89 & 2.80 & 1.97 & 3.06 \\
$I D($ relativistic $)$ & & & & & 0.6 & \\
$m_{\text {tot }}\left(\mu_{B}\right)$ & & & & 2.70 & 4.50 & 5.80 \\
$\mu_{f}$ & 8.71 & 13.68 & 17.03 & 19.95 & 23.08 & 26.16 \\
\hline \hline
\end{tabular}

Thus, there is a significant reduction of the $5 f$ bonding in the compound as compared to the actinide metal, which is due to this band narrowing effect. This volume expansion due to the relative loss of $5 f$ bonding is greater than the volume contraction due to $6 d-5 d$ bonding in the compound. Therefore for systems with itinerant $5 f$ electrons both in the metal and in the intermetallic compound, Vegard's law underestimates the volumes.

Of course, for a quantitative theoretical description of the lattice constants, we require $a b$ initio equation of state calculations and the result of our calculations are shown in Fig. 2 together with the experimental data. Here we have plotted the volumes obtained from a paramagnetic calculation together with the volumes from a spin-polarized and a fully relativistic (but nonpolarized) calculation. The general agreement between experiments and the calculated paramagnetic volumes is good up to $\mathrm{PuIr}_{2}$ where there is a clear difference between the measured and calculated volume. In the next compound, $\mathrm{AmIr}_{2}$, the disagreement is even larger. Neglecting for the moment these two discrepancies we notice that the experimental parabolic trend in volume for the early $A \mathrm{Ir}_{2}$ systems is well reproduced.

To understand this trend, and especially the role the $5 f$ electrons play, we have plotted the self-consistently obtained $5 f$ partial pressure for the $A \mathrm{Ir}_{2}$ systems from a paramagnetic calculation in Fig. 3. These pressures were all taken from a fixed volume (chosen to be $106 \AA^{3}$ ) in the calculations, to consistently observe the trends in the $5 f$

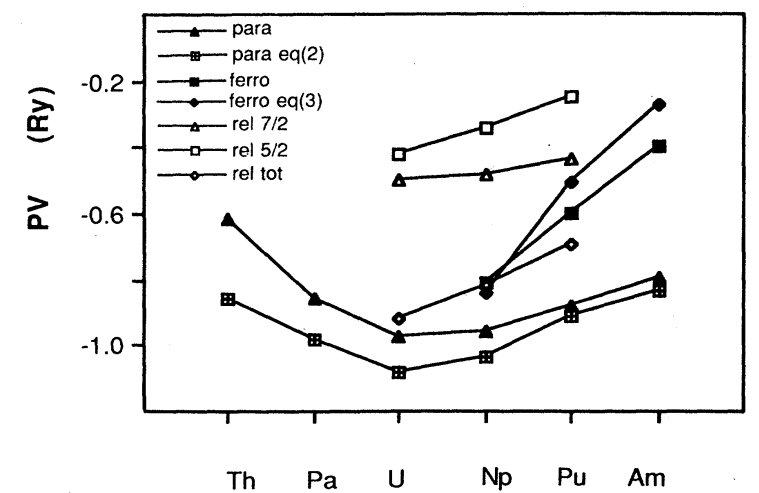

FIG. 3. Actinide $5 f$ partial pressure (scalar relativistic, spinpolarized scalar relativistic, and fully relativistic) for the $A \mathbf{I r}_{2}$ systems at the constant volume $106 \AA^{3}$. bonding. As can be seen from Fig. 3 the paramagnetic $5 f$ pressure indeed shows a parabolic behavior with a maximum contribution for $\mathrm{UIr}_{2}$. This is the result of essentially three effects, namely, (a) the filling of the $5 f$ band, (b) the variation of the $5 f$ bandwidth, and (c) the hybridization with the ligand states. The first effect causes the product $n_{f}\left(14-n_{f}\right)$, in Eq. (1), to have its maximum for $n_{f}=7$ and it is the reason for the parabolic trend in the volumes of the transition metals ${ }^{20,24}$ and the actinide metals. ${ }^{25}$ However, for a fixed volume of the $A \mathrm{Ir}_{2}$ series, incomplete screening of the extra added $5 f$ electron, when the atomic number of the actinide is increased (in going, for example, from $\mathrm{UIr}_{2}$ to $\mathrm{NpIr}_{2}$ ), contracts the $5 f$ wave function and the $5 f$ band becomes more narrow (in Table $I$ this is reflected in the increasing value of the band mass $\mu_{f}$ ). Therefore the maximum in bonding $5 f$ pressure is moved from $n_{f}=7(\mathrm{Cm})$ to somewhere between uranium and neptunium. This is in fact similar to the trend the $5 f$ pressures show in the $A$ metals calculated for a fixed common volume. This second effect is, however, often completely compensated by the band broadening due to the contraction of the lattice when we occupy bonding orbitals. The third effect originates from the hybridization between actinide $5 f$ and ligand $d$ (or $p$ ) states and it has earlier been explained to account for the minimum of the lattice constant at $\mathrm{UN}$ in the $A \mathrm{~N}$ series. ${ }^{12}$

Sometimes it can be useful to calculate the equation of state by means of a more transparent but less accurate expression than Pettifor's full pressure formula. ${ }^{20}$ This is the first-order pressure expression ${ }^{20,22,12,9}$ and we will use it here to ease the interpretation of the different contributions to the chemical bonding. Using this expression the partial pressure divides into a band center term and a bandwidth term and it can be written

$(3 p V)_{1}=n_{1}\left(\frac{-\partial C_{1}}{\partial \ln S}\right)+n_{1}\left(E_{1}-C_{1}\right)\left(-\frac{\partial \ln W_{1}}{\partial \ln S}\right)$.

Here $E_{1}$ is the center of gravity of the occupied part of the 1 band and $C_{1}$ is the center of this band. The $5 f$ partial pressure from Eq. (2) has been calculated and is plotted in Fig. 3. In Eq. (2) the first term (called the band center term) is found to be $0.2 \mathrm{Ry}$ for $\mathrm{ThIr}_{2}$ and to decrease continuously to a value of $0.04 \mathrm{Ry}$ in $\mathrm{AmIr}_{2}$ (Table I). Hence we find that it is the second term (called the bandwidth term) that dominates in these systems and 
that it causes the $5 f$ partial pressure and consequently the volumes to have a parabolic trend (Table I). Also the agreement using Eq. (2) as compared to the full pressure formula ${ }^{20}$ is good.

In order to get a general picture of the band structure for a whole series of compounds it can be illustrative to estimate the bandwidth and band positions using the bottom, center, and top band parameters. ${ }^{18,20}$ These have been plotted in Fig. 4 and give a rough picture of the energy band structure (at the constant volume $106 \AA^{3}$ ). According to this model the electronic structure of $\mathrm{ThIr}_{2}$ consists of a broad $d$ band cut by the Fermi energy $\left(E_{F}\right)$ at the upper part of this band. Above $E_{F}$ there is a fairly broad $5 f$ band (bandwidth $0.35 \mathrm{Ry}$ ), but this band is unoccupied and thorium behaves as a normal tetravalent element like $\mathrm{Ti}, \mathrm{Zr}$, or $\mathrm{Hf}$. In the other compounds of the $A \mathrm{Ir}_{2}$ series both the $d$-band width and the position are more or less invariant. The major changes occur in the $5 f$ band which becomes more narrow and whose energy is lowered relative to $E_{F}$. Hence $\mathrm{PaIr}_{2}$ is the first compound with $5 f$ bands below $E_{F}$ (this is found in the actinide metals as well and also in compounds ${ }^{12,9}$ ) and the $5 f$ bandwidth is $0.2 \mathrm{Ry}$. For the systems $\mathrm{UIr}_{2}$ up to $\mathrm{AmIr}_{2}$ the $5 f$ band is pinned to $E_{F}$ lowering its energy just enough to add approximately one electron per $A$ atom with increasing atomic number. Here the effect of incomplete screening can also be seen since the bandwidth gradually becomes narrower and in $\mathrm{AmIr}_{2}$ its width is reduced to $0.1 \mathrm{Ry}$.

We conclude this section by remarking that the above presented simplified model of the density of states (DOS) is close to those shown in Fig. 5. These latter DOS's are computed from the eigenvalues in the BZ selfconsistently and consequently contain the detailed bandstructure information, especially hybridization effects. These hybridization effects are the reason for the long tail of the $5 f$ DOS which extends quite far into the $5 d$ band, and it is these tails of the DOS that causes the $5 f$ count

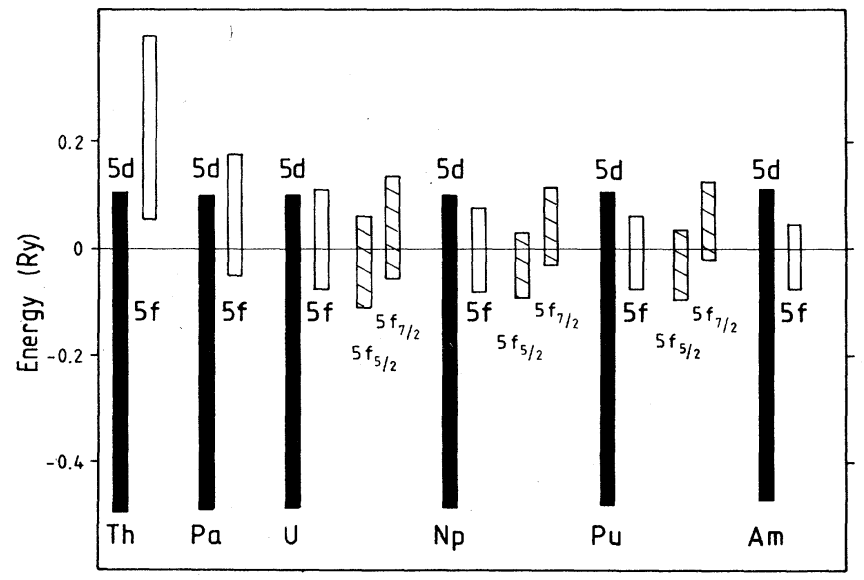

FIG. 4. Schematic picture of the electronic structure of the $A \mathrm{Ir}_{2}$ systems (for details see text). Solid rectangles are the $\operatorname{Ir} d$ states, open rectangles are the $A f$ states, and dashed rectangles are the $A 5 f_{5 / 2}$ and the $A 5 f_{7 / 2}$ states.

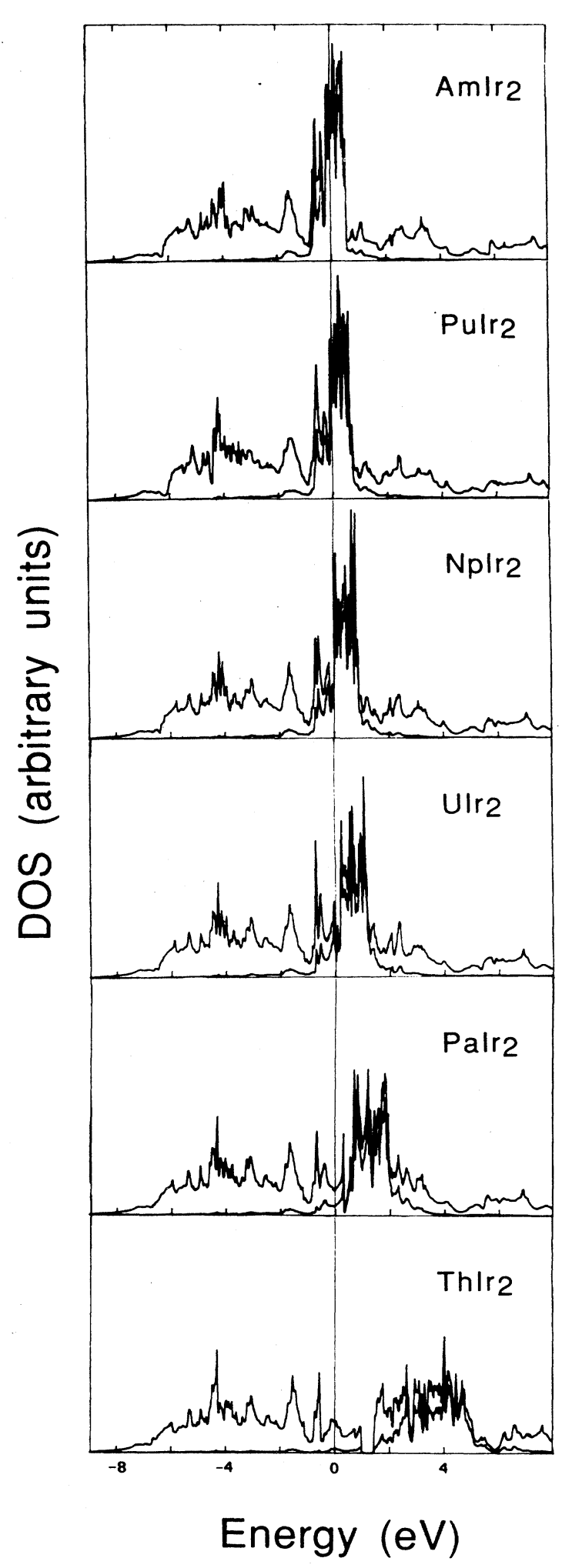

FIG. 5. Calculated densities of states (DOS) for the $A \mathrm{Ir}_{2}$ systems. In each panel the upper curve is the total DOS and the lower is the $A 5 f$ partial DOS. 
to be about 0.8 in $\operatorname{ThIr}_{2}$ instead of zero as in the schematic model above. Otherwise the width of the $5 d$ and $5 f$ bands, as well as their positions, are more or. less the same as in Fig. 4.

\section{SPIN-POLARIZED CALCULATIONS}

The Stoner product $I D\left(E_{F}\right)$ for all compounds [calculated as explained in detail in Ref. 26; $I$ is the Stoner exchange parameter and $D\left(E_{F}\right)$ is the total DOS at the Fermi energy] was calculated for the paramagnetic state. From Table I we notice that for $\mathrm{ThIr}_{2}$ and $\mathrm{PaIr}_{2}$ the Stoner product is low (as is normal for $\mathrm{Th}$ and $\mathrm{Pa}$ and their compounds ${ }^{12,9}$ ) but for $\mathrm{UIr}_{2}$ the Stoner product is 0.89 at the experimental volume. This high value is in full agreement with the suggestion ${ }^{2}$ of spin fluctuations being present in this system.

However, for $\mathrm{NpIr}_{2}$ the Stoner product is larger than one and for a ferromagnetic spin ordering the selfconsistently calculated spin moment in $\mathrm{NpIr}_{2}$ is $2.7 \mu_{B}$ per formula unit. This large moment gives rise to large magnetovolume effects and brings the theoretical lattice constant into rather good agreement with experiment. The absolute value of the magnetic moment is, however, in bad agreement with the experimental value. The reason for this is most likely the large orbital contributions to the total magnetic moment one encounters when dealing with $5 f$ band magnetism. ${ }^{10}$ In the light actinides the orbital moment is coupled antiparallel to the spin moment and therefore it reduces the magnitude of the total moment. Still the large intrinsic spin magnetism reduces the $5 f$ pressure and in order to quantify this reduction we have used Eq. (2) but allowed for spin polarization. The bandwidth term then becomes ${ }^{20,22,12,9}$

$$
(3 p V)_{f}^{\text {pol }}=(3 p V)_{f}^{\text {para }}\left\{1-m_{f}^{2} /\left[14 n_{f}\left(1-n_{f} / 14\right)\right]\right\} \text {, }
$$

where $m_{f}$ is the spin polarization of the $5 f$ electrons. Thus a $5 f$ spin moment will reduce the $5 f$ contribution to the bonding as compared to the paramagnetic state. For $\mathrm{NpIr}_{2}$ this reduces the $5 f$ contribution to the chemical bonding by about $40 \%$ and the theoretical lattice constant from this calculation is actually somewhat larger than the experimental one (Fig. 2). In $\mathrm{PuIr}_{2}$ a substantial $5 f$ spin moment also developed when we allowed for spin polarization (Table I). Finally in $\mathrm{AmIr}_{2}$ the $5 f$ spin moment almost saturates, filling the spin-up band and strongly reducing the $5 f$ bonding pressure. This causes a dramatic change in the chemical bonding of the $5 f$ electrons. It has previously been suggested that a completely spin-polarized situation for a half-filled band system can account for the localization of the $5 f$ electrons $^{27,22,12,9}$ and we make the same interpretation here. Therefore the good agreement between the calculated spin-polarized and the experimental volume in Fig. 2 is consistent with a localized $5 f^{6}$ configuration in $\mathrm{AmIr}_{2}$.

In Fig. 3 we have plotted the partial $5 f$ pressure from the spin-polarized calculations of $\mathrm{NpIr}_{2}, \mathrm{PuIr}_{2}$, and $\mathrm{AmIr}_{2}$ both as obtained from the full pressure formula ${ }^{20}$ and from Eq. (3). Here the large effect the magnetic moment has on the bonding pressure in Eq. (3) becomes clear and when, for a half-filled band, the spin polarization is complete, Eq. (3) gives a zero contribution. The reason we get a nonzero although small pressure from Eq. (3) for $\mathrm{AmIr}_{2}$ can be traced back to the $0.25 f$ electrons being present in the spin-down band. For the spinpolarized calculations, as well as the paramagnetic calculations, the simple model in Eqs. (2) and (3) gives a good understanding of the chemical bonding. We conclude this section by noting that the spin-polarized calculations account very well for the volumes of $\mathrm{NpIr}_{2}$ and $\mathrm{AmIr}_{2}$ but that the moment of the $\mathrm{Np}$ site in $\mathrm{NpIr}_{2}$ is overestimated, although this is most likely mainly due to our neglection of the spin-orbit coupling. The nonmagnetic state of $\mathrm{PuIr}_{2}$ could, however, not be explained in the calculations and this problem will be addressed below.

\section{RELATIVISTIC EFFECTS}

Although the calculations described above contain the important relativistic mass velocity term and the Darwin shift, they do not include the spin-orbit term which is less important for other than magnetic properties. This can however, as we have shown elsewhere, sometimes be too crude an approximation. ${ }^{10,28,29}$ Therefore we shall now describe the results of fully relativistic calculations for $\mathrm{UIr}_{2}, \mathrm{NpIr}_{2}$, and $\mathrm{PuIr}_{2}$. By "fully relativistic" we mean that we solve the Dirac equation and the relativistic band-structure problem self-consistently. ${ }^{30,28}$ The difference between these calculations and the scalarrelativistic calculations is essentially the inclusion of the spin-orbit coupling although that is not explicit in the formalism. The equilibrium volumes obtained from these calculations are plotted in Fig. 2, whereas the partial $5 f$ pressures divided into the $5 f_{5 / 2}$ and $5 f_{7 / 2}$ parts are plotted in Fig. 3. It is clear from Fig. 2 that the change in volume due to spin-orbit coupling is small for $\mathrm{UIr}_{2}$ and $\mathrm{NpIr}_{2}$. In $\mathrm{PuIr}_{2}$, however, the effect is already quite substantial and the calculated volume is actually brought into good agreement with experiment. The reason for this is that in $\operatorname{PuIr}_{2}$ the $5 f_{5 / 2}$ and the $5 f_{7 / 2}$ bands are split apart by spin-orbit coupling so much that the $5 f_{5 / 2}$ occupation number by far exceeds that of the $5 f_{7 / 2}$ band. Consequently the $5 f_{5 / 2}$ band is almost filled whereas the $5 f_{7 / 2}$ band is almost empty, strongly reducing the partial $5 f$ electronic pressure. In order to demonstrate the spinorbit effect more clearly we have in Fig. 4 plotted the estimated bandwidths and band positions from the bottom, center, and top band parameters obtained from the fully relativistic calculations. A convenient measure of the importance of spin-orbit coupling is the ratio $R_{2}=n_{j=1-1 / 2} / n_{j=1+1 / 2} \cdot{ }^{28,9}$ We show the values of this ratio $^{28,9}$ for the pure elements $\mathrm{U}, \mathrm{Np}$, and $\mathrm{Pu}$ together with our present values for $\mathrm{UIr}_{2}, \mathrm{NpIr}_{2}$, and $\mathrm{PuIr}_{2}$ in Fig. 6. Here it can be seen that the effect of spin-orbit coupling is much larger in the $A \mathrm{Ir}_{2}$ compounds than for the pure actinide metals, which is due to narrower $5 f$ bands in the compounds. It can also be seen that for the $\mathrm{PuIr}_{2}$ system this ratio becomes very large, demonstrating strong effects of spin-orbit coupling for this compound.

We now turn to the interesting nonmagnetic state of PuIr $_{2}$ which we could not account for by using the scalar relativistic calculations. In this case the computed Stoner product was 1.9 and consequently the system will become spin polarized. In the same manner as described else- 


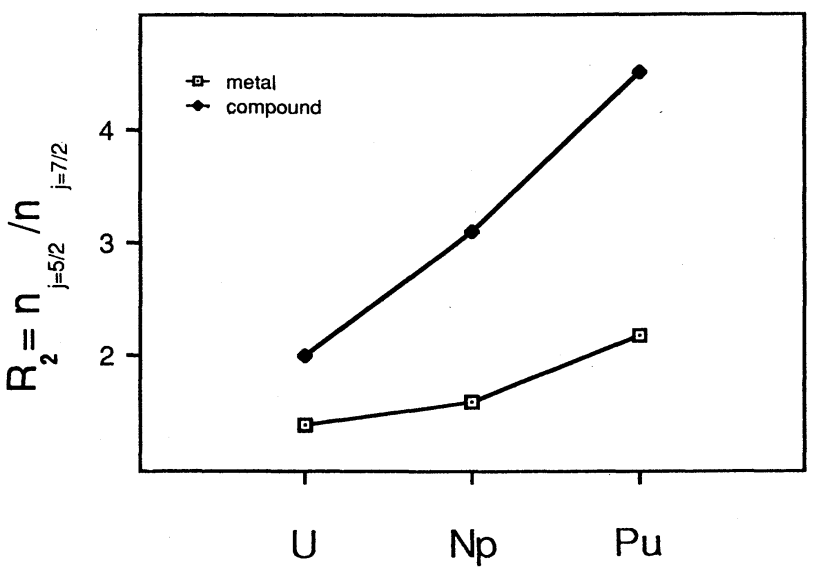

FIG. 6. Calculated ratio between the $A 5 f_{5 / 2}$ and the $A$ $5 f_{7 / 2}$ occupation numbers for the $A \operatorname{Ir}_{2}$ systems and the $A$ metals (Ref. 28).

where ${ }^{31}$ we obtained the value of the relativistic Stoner product from the fully relativistic calculation. This value is 0.6 , which is a dramatic reduction as compared to normal multiband Stoner theory. ${ }^{26}$ Hence the strong spinorbit coupling in $\mathrm{PuIr}_{2}$ suppresses magnetism and ex-

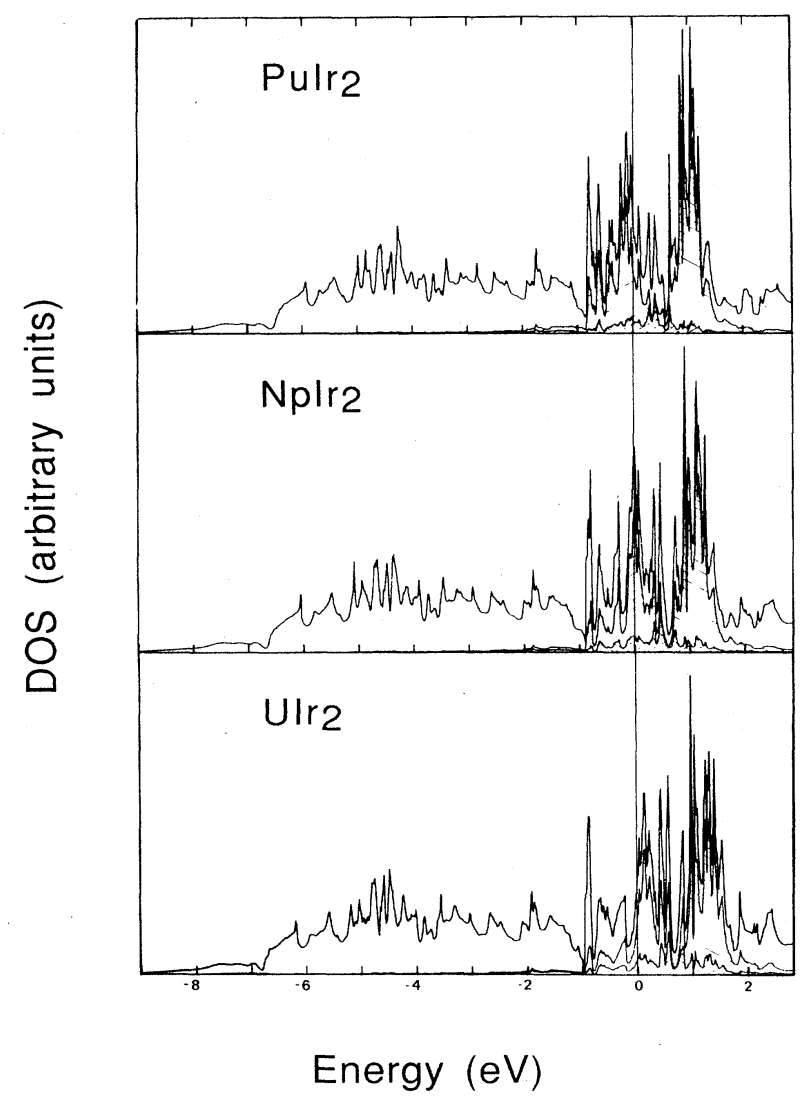

FIG. 7. Calculated total, $A 5 f_{5 / 2}$ and $A 5 f_{7 / 2}$ DOS for the $A \mathrm{Ir}_{2}$ systems. The $5 f_{5 / 2}$ and $5 f_{7 / 2}$ bands are hatched by differently tilted lines, with the $5 f_{7 / 2}$ band being highest in energy. plains the observed temperature-independent paramagnetism. This effect was earlier argued to account for the nonmagnetic state of $\delta$-Pu (Ref. 32) and also here it accounts for the nonmagnetic state of $\mathrm{PuIr}_{2}$. In earlier calculations of this type ${ }^{31}$ we have found that it is for systems where the spin-orbit coupling creates a dramatic reduction of the DOS at the Fermi level that a large reduction of the Stoner product takes place. In the most extreme cases, the spin-orbit splitting of the $5 f$ band can even give rise to an energy gap and be accompanied by semiconducting properties, whereas a scalar relativistic treatment would predict spin ordering. ${ }^{29}$ Examining the DOS at the Fermi level for the $A \operatorname{Ir}_{2}$ systems we find that it is only for $\mathrm{PuIr}_{2}$ that a substantial reduction takes place and therefore we have only performed the cumbersome calculation of the relativistic Stoner product for this compound. Finally, in Fig. 7, we have plotted the DOS from our fully relativistic calculation for $\mathrm{UIr}_{2}$, $\mathrm{NpIr}_{2}$, and $\mathrm{PuIr}_{2}$. Here as well as in Fig. 4 and Fig. 6 the effect of the spin-orbit coupling is clear and the $5 f_{5 / 2}$ and the $5 f_{7 / 2}$ states are rather well separated. The spin-orbit splitting in $\mathrm{UIr}_{2}$ is $0.9 \mathrm{eV}$, in $\mathrm{NpIr}_{2}$ it is $1.0 \mathrm{eV}$, and finally it is $1.2 \mathrm{eV}$ in $\mathrm{PuIr}_{2}$. This is comparable to the actinide $5 f$ bandwidths in these systems. Apart from these spinorbit splittings the similarity with the scalar-relativistic calculations is striking (Fig. 5).

\section{MODEL CALCULATION FOR THE $\operatorname{AIr}_{2}$ SYSTEMS}

We will now describe a more detailed semianalytic investigation of the different contributions to the band structure in the present compounds. Such an exercise is especially interesting in these systems since the direct overlap between the $5 f$ wave functions is quite large and at the same time hybridization effects are substantial.

The bandwidth can, neglecting hybridization, be written as $^{22,11,12,9}$

$$
W_{t l}=\left[\left(12 / N_{t l}\right) S_{t l}^{t l 2}\right]^{1 / 2} \Delta_{t l} .
$$

Here $\Delta_{t l}$ is

$$
\Delta_{t l}=1 /\left[\mu_{t l} s_{t}^{2}\left(s / s_{t}\right)^{2 l+1}\right]
$$

and $N_{t l}=(2 l+1) n_{t}$, where $n_{t}$ is the number of type $t$ atoms per primitive cell and 1 is the angular momentum. Furthermore $S_{t l}^{t l 2}$ is the second moment of the canonical $t l$ band, $s_{t}$ is the atomic radius of atom $t$, and $s$ is a characteristic radius for the lattice. ${ }^{22,11,12,9,33}$ Using these expressions and evaluating moments for the cubic Laves phase, an estimate of the canonical bandwidths of, for example, the $\mathrm{UIr}_{2}$ compound yields a $0.5 \mathrm{Ry}$ broad Ir $d$ band and a $\mathrm{U} f$ band of width $0.1 \mathrm{Ry}$. This $\mathrm{Ir} d$ bandwidth is in excellent agreement with the ones calculated including hybridization (Fig. 5). For the U $5 f$ states, however, only $50 \%$ of the total bandwidth can be accounted for, when hybridization is neglected. Hence hybridization with the Ir $d$ states is of equal importance for the width of the $5 f$ states as the direct overlap between the $5 f$ wave functions. A good measure of the hybridization is the number of $t l$ electrons that reside in what unhybridized is a pure $t^{\prime} l^{\prime}$ band, i.e., $N_{t l}^{t^{\prime} l^{\prime}}$. This can be calculated using 
TABLE II. Second moments of the structure constants for the Laves phase structure. Also given is the Ir $d$ band mass and the number of actinide $5 f$ electrons residing in the iridium $5 d$ band, calculated from Eq. (6).

\begin{tabular}{lc}
\hline$S_{\mathrm{Ir} d}^{\mathrm{Ir} d^{2}}$ & 1180 \\
$S_{\mathrm{U} f^{2}}^{\mathrm{U}}$ & 80 \\
$S_{\operatorname{Ir} d}^{\mathrm{U} f^{2}}$ & 264 \\
$\mu_{\mathrm{Ir} d}$ & 4.6 \\
$N_{\mathrm{Ir} d}^{\mathrm{Th} f}$ & 0.91 \\
$N_{\mathrm{Ir} d}^{\mathrm{Pa}}$ & 0.74 \\
$N_{\mathrm{Ir} d}^{\mathrm{U} f}$ & 1.1 \\
$N_{\mathrm{Ir} d}^{\mathrm{Np} f}$ & 1.2 \\
$N_{\mathrm{Ir} d}^{\mathrm{Pu} f}$ & 1.2 \\
\hline \hline
\end{tabular}

$$
N_{t l}^{t^{\prime} l^{\prime}}=2 S_{t l}^{t^{\prime} l^{\prime 2}} \Delta_{t l} \Delta_{t^{\prime} l^{\prime}}\left(C_{t l}-C_{t^{\prime} l^{\prime}}\right)^{-2}
$$

Here $C_{t l}$ and $C_{t^{\prime} l^{\prime}}$ are the center of bands for the $t l$ and $t^{\prime} l^{\prime}$ band, respectively. Armed with Eq. (6) we can now easily calculate the strength of the hybridization, i.e., the number of actinide $5 f$ electrons that are hybridized into an originally pure iridium $5 d$ band, for the different compounds in the $A \mathrm{Ir}_{2}$ systems. These values are given in Table II, and we notice that the $0.85 f$ electrons found in $\mathrm{ThIr}_{2}$ are entirely located in what was originally a pure Ir $5 d$ band. When the actinide atomic number increases the difference between $C_{f}$ and $C_{d}$ decreases and consequently $N_{\text {Ir } d}^{A f}$ is expected to increase. However $\Delta_{f}$ is decreasing along the series and this decreases $N_{\mathrm{Ir} d}^{A} f$. Since these two effects to a large extent cancel this value is almost constant from $\mathrm{UIr}_{2}$ to $\mathrm{PuIr}_{2}$ (Table II). Also hybridization pushes the two pure $t l$ and $t^{\prime} l^{\prime}$ bands apart by an amount

$$
\delta E_{t l}=\frac{1}{N_{t l}} S_{t l}^{t^{\prime} l^{\prime} 2} \Delta_{t l} \Delta_{t^{\prime} l^{\prime}}\left(C_{t l}-C_{t^{\prime} l^{\prime}}\right)^{-1}
$$

Inserting appropriate numbers, for instance, for $\mathrm{UIr}_{2}$ we find that the iridium $5 d$ band is pushed down by $6 \mathrm{mRy}$ and the uranium $5 f$ band is pushed up by $8 \mathrm{mRy}$.

\section{CONCLUSION}

By using $a b$ initio energy band calculations we have obtained a detailed picture of the electronic structure, magnetic properties, and equilibrium volumes for the $A \mathrm{Ir}_{2}$ systems. Good agreement was found both for the occurrence of magnetism and the equilibrium volumes. In accordance with experiment we find that $\mathrm{NpIr}_{2}$ is the only compound that orders magnetically. The paramagnetism of $\mathrm{PuIr}_{2}$ can only be understood from a fully relativistic treatment. Special attention to hybridization effects have also been given and it is shown that the $5 f$ bandwidth originates almost equally from the $5 f$ wave function overlap as from hybridization between the $5 f$ and the ligand $5 d$ states.

Also the problem of localization has been addressed by means of spin-polarized calculations, where an almost completely spin-polarized and close to half-filled band situation was found in $\mathrm{AmIr}_{2}$. We ascribe this to a localized $5 f^{6}$ ground state in $\mathrm{AmIr}_{2}$ and this compound is consequently the first localized $A \mathrm{Ir}_{2}$ system. It is interesting to note that the calculations reproduce the experimentally observed volume jump between $\mathrm{PuIr}_{2}$ and $\mathrm{AmIr}_{2}$. This is also the case for the pure actinides with a dramatic volume change between $\mathrm{Pu}$ and $\mathrm{Am} .^{25,27}$ This success of the local-density approximation (LDA) seems to be in contrast to the rather poor results for conventional mixed valence systems like $\mathrm{SmS} .{ }^{34}$ The main difference is that for the present actinide systems the change is from a bonding to a nonbonding situation for the $5 f$ electrons, while the mixed valence in $\mathrm{SmS}$ involves two different nonbonding configurations $4 f^{6}$ and $4 f^{5}$. The difficulty to simultaneously deal with two localized configurations is probably the reason why LDA calculations so far have met with rather limited success for conventional mixed valence systems.

\section{ACKNOWLEDGMENTS}

O. Eriksson is grateful to The Bank of Sweden Tercentenary Foundation and B. Johansson is grateful to the Swedish Natural Science Research Council for financial support. Valuable discussions with Dr. V. Sechovsky' and Dr. L. Havela are acknowledged.
*Permanent address: Condensed Matter Theory Group, Department of Physics, University of Uppsala, Box 530, S75121 Uppsala, Sweden.

${ }^{1}$ B. I. Matthias, V. B. Compton, and E. Corenzwit, J. Phys. Chem. Solids 19, 130 (1961).

${ }^{2}$ V. Sechovský and L. Havela, in Intermetallic Compounds of Actinides, Vol. 4 of The Handbook of Ferromagnetic Materials, edited by E. P. Wohlfarth and K. H. J. Buschow (NorthHolland, Amsterdam, 1988).

${ }^{3}$ M. B. Brodsky and R. J. Trainor, Bull. Am. Phys. Soc. 21, 442 (1976).

4J. Gal, Z. Hadari, U. Atzmony, E. R. Bauminger, I. Nowik, and S. Ofer, Phys. Rev. B 8, 1901 (1973).

${ }^{5}$ A. I. Aldred, B. D. Dunlap, D. J. Lam, and I. Nowik, Phys. Rev. B 10, 1011 (1974).
${ }^{6}$ M. B. Brodsky and R. J. Trainor, J. Phys. (Paris) Colloq. 8, C6-777 (1978).

${ }^{7}$ W. J. Nellis and M. B. Brodsky, in Magnetism and Magnetic Materials (Chicago, 1971), Proceedings of the 17th Annual Conference on Magnetism and Magnetic Materials, AIP Conf. Proc. No. 5, edited by C. D. Graham, Jr. and J. J. Rhyne (AIP, New York, 1972).

${ }^{8}$ A. R. Harvey, M. B. Brodsky, and W. J. Nellis, Phys. Rev. B 7, 4137 (1973).

${ }^{9}$ M. S. S. Brooks, B. Johansson, and H. L. Skriver, in Handbook on the Physics and Chemistry of the Actinides, edited by A. J. Freeman and G. H. Lander (North-Holland, Amsterdam, 1984), Vol. 1, p. 153.

${ }^{10}$ M. S. S. Brooks and P. J. Kelly, Phys. Rev. Lett. 51, 1708 (1983); M. S. S. Brooks, O. Eriksson, B. Johansson, J. J. M. 
Franse, and P. H. Frings, J. Phys. F 18, 133 (1988); M. R. Norman and D. D. Koelling, Phys. Rev. B 33, 3803 (1986).

${ }^{11}$ M. S. S. Brooks and D. Glötzel, Physica B + C 102B, 51 (1980); J. Magn. Magn. Mater. 15-18, 873 (1980).

${ }^{12}$ M. S. S. Brooks, J. Phys. F 14, 639 (1984); 14, 653 (1984); 14, 657 (1984).

${ }^{13}$ D. D. Koelling, B. D. Dunlap, and G. W. Crabtree, Phys. Rev. B 31, 4966 (1984).

${ }^{14}$ R. C. Albers, Phys. Rev. B 32, 7646 (1985).

${ }^{15}$ B. Johansson, O. Eriksson, M. S. S. Brooks, and H. L. Skriver, Phys. Scr. T13, 65 (1986); Inorgan. Chim. Acta 140, 59 (1987); O. Eriksson, B. Johansson, M. S. S. Brooks, and H. L. Skriver, Phys. Rev. B 38, 12858 (1988).

${ }^{16}$ A. J. Freeman and D. D. Koelling, in The Actinides: Electronic Structure and Related Properties, edited by A. J. Freeman and J. B. Darby (Academic, New York, 1974), Vol. I, p. 51.

${ }^{17}$ H. H. Hill, Plutonium 1970 and Other Actinides, edited by W. N. Miner (The Metallurgical Society of the AIME, New York, 1970), p. 2.

${ }^{18}$ O. K. Andersen, Phys. Rev. B 12, 3060 (1975); H. L. Skriver, The LMTO Method (Springer-Verlag, Berlin, 1984).

${ }^{19}$ U. von Barth and L. Hedin, J. Phys. C 5, 1629 (1972).

${ }^{20}$ D. G. Pettifor, Commun. Phys. 1, 1 (1976); A. R. Mackintosh and O. K. Andersen, in Electrons at the Fermi Surface, edited by M. Spingford (Cambridge University Press, Cambridge, England, 1979); O. K. Andersen, O. Jepsen, and D. Glötzel, in Highlights in Condensed Matter Theory, edited by F. Bassani, F. Fumi, and M. P. Tosi (North-Holland, New York,
1985).

${ }^{21}$ L. Vegard, Z. Phys. 5, 17 (1921).

${ }^{22}$ O. K. Andersen, H. L. Skriver, H. Nohl, and B. Johansson, Pure Appl. Chem. 52, 93 (1979).

${ }^{23}$ O. Eriksson, B. Johansson, H. L. Skriver, and M. S. S. Brooks, Physica B + C 144B, 32 (1986).

${ }^{24}$ V. L. Moruzzi, A. R. Williams, and J. F. Janak, Phys. Rev. B 15, 2854 (1977); D. G. Pettifor, J. Phys. F 8, 219 (1978).

${ }^{25}$ H. L. Skriver, O. K. Andersen, and B. Johansson, Phys. Rev. Lett. 48, 42 (1978).

${ }^{26}$ S. H. Vosko and J. P. Perdew, Can. J. Phys. 53, 1385 (1975); J. F. Janak, Phys. Rev. B 16, 255 (1977); M. S. S. Brooks, O. Eriksson, and B. Johansson, Phys. Scr. 35, 52 (1987).

${ }^{27}$ H. L. Skriver, O. K. Andersen, and B. Johansson, Phys. Rev. Lett. 44, 1230 (1980).

${ }^{28}$ M. S. S. Brooks, J. Phys. F 13, 103 (1983).

${ }^{29}$ M. S. S. Brooks, J. Magn. Magn. Mater. 63/64, 649 (1987).

${ }^{30}$ N. E. Christensen, J. Phys. F 8, L51 (1978); C. Godreche, J. Magn. Magn. Mater. 29, 262 (1982).

${ }^{31}$ O. Eriksson, M. S. S. Brooks, and B. Johansson, J. Phys. (Paris) (to be published).

${ }^{32}$ M. S. S. Brooks, B. Johansson, O. Eriksson, and H. L. Skriver, Physica B + C 144B, 1 (1986).

${ }^{33}$ The characteristic radius of the lattice, which is used in Eqs. (4) and (5), was chosen to be the average radius per formula unit. Normally it is chosen as the radius of the sublattice.

${ }^{34}$ Z. W. Lu, D. J. Singh, and H. Krakauer, Phys. Rev. B 37, 10045 (1988). 